\section{Carbon suboxide in comet Halley}

SIR - Huntress et al. suggested ${ }^{1}$ that carbon suboxide $\left(\mathrm{C}_{3} \mathrm{O}_{2}\right)$ could exist in the nucleus of comet Halley, and that this species would be the progenitor of atomic carbon and carbon monoxide present in the cometary atmosphere. A $\mathrm{C}_{3} \mathrm{O}_{2}$ production rate of $0.03-0.04$ times that of water would explain the observed

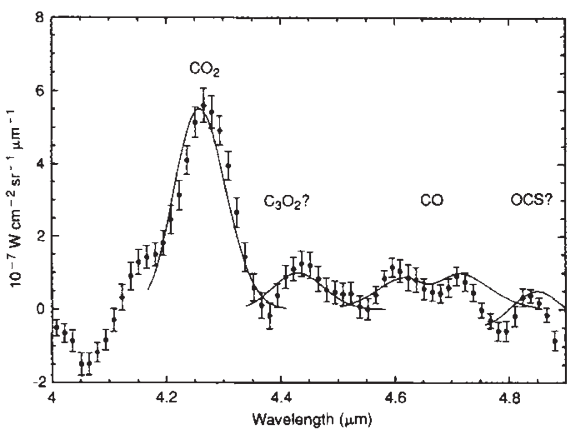

Reproduction of a part of the spectrum of comet Halley observed by the infrared spectrometer aboard the VEGA 1 probe $^{4}$. Solid lines, synthetic band profiles for $\mathrm{CO}_{2}, \mathrm{C}_{3} \mathrm{O}_{2}$, $\mathrm{CO}$ and OCS.

abundances and distributions of cometary $\mathrm{C}$ and $\mathrm{CO}$. Huntress et al. ${ }^{1}$ planned to look for $\mathrm{C}_{3} \mathrm{O}_{2}$ evidence in the Giotto mass-spectrometer data.

Another way to assess the presence of cometary $\mathrm{C}_{3} \mathrm{O}_{2}$ is to look for its infrared vibrational bands. Its $v_{3}$ band at $4.43 \mu \mathrm{m}$ is very strong ${ }^{2}$ (band strength of 8,900 $\mathrm{cm}^{-2} \mathrm{~atm}^{-1}$ ). The expected fluorescence rate of this band, at $1 \mathrm{AU}$ from the Sun, is $1.0 \times 10^{-2} \mathrm{~s}^{-1}$, compared to $2.9 \times$ $10^{-3}$ for the $v_{3}$ band of $\mathrm{CO}_{2}$ at $4.25 \mu \mathrm{m}$ and to $2.6 \times 10^{-4}$ for the $v_{3}$ band of $\mathrm{H}_{2} \mathrm{O}$ at $2.7 \mu \mathrm{m}$. If $\mathrm{C}_{3} \mathrm{O}_{2}$ had a mixing ratio of a few per cent, comparable to the mixing ratio of $\mathrm{CO}_{2}$, its $v_{3}$ band would be stronger than the $v_{3}$ band of $\mathrm{CO}_{2}$ and very conspicuous in infrared cometary spectra.

We measured 2.5-5.0- $\mu \mathrm{m}$ spectra in comet Halley with the IKS experiment aboard the VEGA 1 probe $^{3,4}$. In addition to water, $\mathrm{CO}_{2}, \mathrm{CO}$ and $\mathrm{H}_{2} \mathrm{CO}$ were detected with mixing ratios of $2.7 \%, 5 \%$ and $4 \%$, respectively. OCS was tentatively detected with a mixing ratio of $0.7 \%$ or less. The figure shows that the $v_{3}$ band of $\mathrm{CO}_{2}$ is conspicuous, but that a weak feature of $4.45 \mu \mathrm{m}$ may also be present. This must not be considered as a definite detection of $\mathrm{C}_{3} \mathrm{O}_{2}$, as the feature may also be identified in other species (possibly $\mathrm{C} \equiv \mathrm{N}$ groups; ref. 4). If the feature is indeed due to $\mathrm{C}_{3} \mathrm{O}_{2}$, its intensity corresponds to an abundance $\left[\mathrm{C}_{3} \mathrm{O}_{2}\right] /\left[\mathrm{H}_{2} \mathrm{O}\right]=0.0011$ (see ref. 4 for method of calculation). This can be considered as an upper limit to the cometary abundance of $\mathrm{C}_{3} \mathrm{O}_{2}$ directly ejected from the nucleus.
Although the presence of a distributed source of $\mathrm{C}_{3} \mathrm{O}_{2}$ cannot be excluded, infrared observations show that parent $\mathrm{C}_{3} \mathrm{O}_{2}$, if present, is less abundant than speculated by Huntress $e t$ al.

JACQUES CROVISIER

THÉRĖSE ENCRENAZ

MICHEL COMBES

Observatoire de Paris-Meudon,

\section{F-92195 Meudon, France}

1. Huntress, W. T. Jr, Allen, M. \& Delitsky, M. Nature 352 316-318 (1991)

2. Pugh, L. A. \& Rao, K. N. in Molecular Spectroscopy Modern Research (ed. Rao, K. N.) Vol. II 165-227 (Academic, London, 1976)

3. Combes, M. et al. Nature 321, 266-268 (1986).

4. Combes, M. et al. icarus 76, 404-436 (1988).

\section{Tests for rodent polyphyly}

SIR - Graur et al. suggested $^{1}$ that the order Rodentia may not be monophyletic, with guinea-pigs and their relatives being outside a clade consisting of Primates, Artiodactyla and the rodent suborder Sciurognathi. They argue that the recognition of the guinea-pig and relatives as a separate order of mammals "may resolve or ameliorate many of the paradoxes associated with the evolution of guinea-pig genes", such as parallel changes in guinea-pigs and birds at the molecular level and accelerated rates of gene evolution. Acceptance of this hypothesis requires the reinterpretation of many morphological characters as homoplastic between the two recognized suborders of Rodentia.

The order Rodentia represents a group of gnawing mammals that combine several morphological characters associated with specializations of the incisors, molar teeth and musculoskeletal features of the masticatory apparatus ${ }^{2}$. Virtually all character systems examined have supported rodent monophyly, including those as diverse as the postcranial skeleton, placental and fetal membranes, carotid arterial patterns and myology $y^{3}$. Although parallelism within the order may make determining relationships among families difficult, from a morphological perspective rodents constitute a distinct group of eutherian mammals and any taxonomic controversy has previously not included a question of their monophyly ${ }^{3}$. A hypothesis of rodent polyphyly therefore replaces the 'molecular paradox' resolved by Graur $e t$ al. with the problem of reinterpreting well-established morphological synapomorphies as homoplasies.

Rodent polyphyly, therefore, challenges what is known about the comparative morphology of this order. Resolution of this conflict between molecular and morphological data is critical for understanding the evolution and phylogenetic use of these different character systems. We have looked at nucleotide sequence data from the mitochondrial $12 \mathrm{~S}$ ribosomal RNA gene for some of the taxa examined by Graur et al. ${ }^{1}$. Only transversions were examined as these have been reported to change linearly with time throughout the eutherian radiation $^{4}$ and for even older divergences $^{5}$ and are least likely to be affected by multiple mutations. The most conservative of these transversions, those occurring in a ditypic state, were analysed separately.

We conducted two levels of phylogenetic analysis, the first to determine relationships among the eutherian orders and to illustrate the value of mitochondrial 12S rRNA gene sequences at the higher hierarchical levels, and the second to assign a root. In the first, both total and ditypic transversions support the arrangement grouping the two rodent suborders separate from the other represented mammalian orders (see figure). The support for this arrangement is significant at $P<0.05$ for both total and ditypic transversions according to a binomial test ${ }^{6}$. Graur et al. also support this arrangement,

a, Three possible dichotomous arrangements of four mammals. The values indicate the number of informative transversions, and the number of ditypic transversions (in parentheses) supporting each arrangement. A ditypic transversion is defined as a sequence position with two species sharing the same purine, and the other two sharing the same pyrimidine $^{7}$. Species abbre-

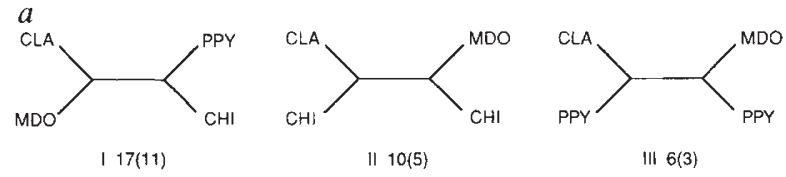

viations are as in $b$. $b$, Best tree found following Graur et al.'s four-taxon method with chicken as the designated outgroup. Ditypic transversions were used solely to compensate for the greater divergence between chicken and our mammalian ingroup. Due to difficulties in determining an optimal alignment, the most variable region of domain II, $5^{\prime}$ of helix 14 , was omitted in all cases. All parsimony analyses were conducted with the PAUP program (version 3.OK, D. L. Swofford). Published sequences available from GenBank. 